Article

\title{
Solutions Modulo $p$ of Gauss-Manin Differential Equations for Multidimensional Hypergeometric Integrals and Associated Bethe Ansatz
}

\author{
Alexander Varchenko \\ Department of Mathematics, University of North Carolina at Chapel Hill, Chapel Hill, NC 27599-3250, USA; \\ anv@email.unc.edu \\ Academic Editor: Laurentiu-George Maxim \\ Received: 22 September 2017; Accepted: 13 October 2017; Published: 17 October 2017
}

\begin{abstract}
We consider the Gauss-Manin differential equations for hypergeometric integrals associated with a family of weighted arrangements of hyperplanes moving parallel to themselves. We reduce these equations modulo a prime integer $p$ and construct polynomial solutions of the new differential equations as $p$-analogs of the initial hypergeometric integrals. In some cases, we interpret the $p$-analogs of the hypergeometric integrals as sums over points of hypersurfaces defined over the finite field $\mathbb{F}_{p}$. This interpretation is similar to the classical interpretation by Yu. I. Manin of the number of points on an elliptic curve depending on a parameter as a solution of a Gauss hypergeometric differential equation. We discuss the associated Bethe ansatz.
\end{abstract}

Keywords: Gauss-Manin differential equations; multidimensional hypergeometric integrals; Bethe ansatz

\section{Introduction}

We consider an arrangement of affine hyperplanes $\left(H_{j}\right)_{j=1}^{n}$ in $\mathbb{C}^{k}$. Let $f_{j}\left(t_{1}, \ldots, t_{k}\right)$ be a first-degree polynomial on $\mathbb{C}^{k}$ whose kernel is $H_{j}$. Let $\left(a_{j}\right)_{j=1}^{n}, \kappa$ be nonzero complex numbers. An associated multidimensional hypergeometric integral is an integral of the form:

$$
I=\int_{\gamma} \prod_{j=1}^{n} f_{j}\left(t_{1}, \ldots, t_{k}\right)^{\frac{a_{j}}{\kappa}} d t_{1} \wedge \cdots \wedge d t_{k}
$$

where $\gamma$ is a cycle in the complement to the union of the hyperplanes. We assume that the hyperplanes depend on parameters $z_{1}, \ldots, z_{n}$ and move parallel to themselves when the parameters change. Then the integral extends to a multivalued holomorphic function of the parameters. The holomorphic function is called a multidimensional hypergeometric function and is associated with this family of arrangements. The simplest example of such a function is the classical hypergeometric function.

The multidimensional hypergeometric functions can be combined into collections so that the functions of a collection satisfy a system of first-order linear differential equations called the Gauss-Manin differential equations.

If all polynomials $\left(f_{j}\right)_{j=1}^{n}$ have integer coefficients and the numbers $\left(a_{j}\right), \kappa$ are integers, then the Gauss-Manin differential equations can be reduced modulo a prime integer $p$ large enough. The goal of this paper is to construct polynomial solutions of the Gauss-Manin differential equations over the field $\mathbb{F}_{p}$ with $p$ elements. Our solutions are $p$-analogs of the multidimensional hypergeometric integrals. The construction of the solutions is motivated by the classical paper [1] by Yu. I. Manin (cf. section "Manin's Result: The Unity of Mathematics" in [2]; see also [3,4]).

The paper is organized as follows. In Section 2, we recall the basic notions associated with an affine arrangement of hyperplanes in $\mathbb{C}^{k}$. In Section 3, we consider a family of arrangements of hyperplanes 
in $\mathbb{C}^{k}$ whose hyperplanes move parallel to themselves when the parameters of the family change. We introduce the Gauss-Manin differential equations and multidimensional hypergeometric integrals. We show that the multidimensional hypergeometric integrals satisfy the Gauss-Manin differential equations (see Theorem 3). In Section 4, we consider the reduction of this situation modulo $p$ and construct polynomial solutions of the Gauss-Manin differential equations over $\mathbb{F}_{p}$ (see Theorem 5), which is the main result of this paper. We interpret our solutions as integrals over $\mathbb{F}_{p}^{k}$ under certain conditions (see Theorem 6). Such integrals could be considered as $p$-analogs of the multidimensional hypergeometric integrals. In Section 5, we consider examples. Under certain conditions, we interpret our polynomial solutions as sums over points on some hypersurfaces over $\mathbb{F}_{p}$ (see Theorem 10). This statement is analogous to the interpretation in Manin's paper [1] of the number of points on an elliptic curve depending on a parameter as a solution of a Gauss hypergeometric differential equation. In Section 6, we briefly discuss the associated Bethe ansatz. We introduce a system of the Bethe ansatz equations and construct a common eigenvector to geometric Hamiltonians out of every solution of the Bethe ansatz equations (see Theorem 11). We show that the Bethe eigenvectors corresponding to distinct solutions are orthogonal with respect to the associated symmetric contravariant form (see Corollary 3).

\section{Arrangements}

We recall some facts about hyperplane arrangements, Orlik-Solomon algebras and flag complexes from [5].

\subsection{An Affine Arrangement}

Let $k$ and $n$ be positive integers, where $k<n$. Denote $J=\{1, \ldots, n\}$.

Let $\mathcal{C}=\left(H_{j}\right)_{j \in J}$, be an arrangement of $n$ affine hyperplanes in $\mathbb{C}^{k}$. Denote $U=\mathbb{C}^{k}-\cup_{j \in J} H_{j}$ as the complement. An edge $X_{\alpha} \subset \mathbb{C}^{k}$ of the arrangement $\mathcal{C}$ is a nonempty intersection of some hyperplanes of $\mathcal{C}$. Denote by $J_{\alpha} \subset J$ the subset of indices of all hyperplanes containing $X_{\alpha}$. Denote $l_{\alpha}=\operatorname{codim}_{\mathbb{C}^{k}} X_{\alpha}$.

We always assume that the arrangement $\mathcal{C}$ is essential; that is, $\mathcal{C}$ has a vertex, an edge that is a point.

An edge is called dense if the subarrangement of all hyperplanes containing it is irreducible: the hyperplanes cannot be partitioned into nonempty sets, so that, after a change of coordinates, hyperplanes in different sets are in different coordinates. In particular, each hyperplane of $\mathcal{C}$ is a dense edge.

\subsection{Flag Complex}

For $\ell=0, \ldots, k$, let $\operatorname{Flag}^{\ell}(\mathcal{C})$ denote the set of all flags:

$$
\mathbb{C}^{k}=L^{0} \supset L^{1} \supset \cdots \supset L^{\ell}
$$

where each $L^{j}$ is an edge of $\mathcal{C}$ of codimension $j$. Let $\mathcal{F}^{\ell}(\mathcal{C}, \mathbb{Z})$ denote the quotient of the free abelian group on $\mathrm{Flag}^{\ell}(\mathcal{C})$ by the following relations. For every flag with a gap:

$$
\widehat{F}=\left(L^{0} \supset L^{1} \supset L^{i-1} \supset L^{i+1} \supset \cdots \supset L^{k}\right), \quad i<k
$$

We impose:

$$
\sum_{F \supset \widehat{F}} F=0
$$

in $\mathcal{F}^{\ell}(\mathcal{C}, \mathbb{Z})$, where the sum is over all flags $F=\left(\widetilde{L}^{0} \supset \widetilde{L}^{1} \supset \ldots \widetilde{\supset} L^{\ell}\right) \in \operatorname{Flag}^{\ell}(\mathcal{C})$, such that $\widetilde{L}^{j}=L^{j}$ for all $j \neq i$. The abelian group $\mathcal{F}^{\ell}(\mathcal{C}, \mathbb{Z})$ is a free abelian group (see [5], Theorem 2.9.2).

There is an "extension of flags" differential $d: \mathcal{F}^{\ell}(\mathcal{C}, \mathbb{Z}) \rightarrow \mathcal{F}^{\ell+1}(\mathcal{C}, \mathbb{Z})$ defined by:

$$
d\left(L^{0} \supset L^{1} \supset \cdots \supset L^{\ell}\right)=\sum_{L^{\ell+1}}\left(L^{0} \supset L^{1} \supset \cdots \supset L^{\ell} \supset L^{\ell+1}\right)
$$


where the sum is over all edges $L^{\ell+1}$ of $\mathcal{C}$ of codimension $\ell+1$ contained in $L^{\ell}$. It follows from Equation (1) that $d^{2}=0$. Thus we have a complex, the flag complex, $(\mathcal{F} \bullet(\mathcal{C}, \mathbb{Z}), d)$.

\subsection{Orlik-Solomon Algebra}

Define abelian groups $\mathcal{A}^{\ell}(\mathcal{C}, \mathbb{Z}), \ell=0,1, \ldots, k$ as follows. For $\ell=0$, set $\mathcal{A}^{0}(\mathcal{C}, \mathbb{Z})=\mathbb{Z}$. For $\ell>0$, $\mathcal{A}^{\ell}(\mathcal{C}, \mathbb{Z})$ is generated by $\ell$-tuples $\left(H_{1}, \ldots, H_{\ell}\right)$ of hyperplanes $H_{i} \in \mathcal{C}$, subject to the relations:

(i) $\left(H_{1}, \ldots, H_{\ell}\right)=0$ if $H_{1}, \ldots, H_{\ell}$ are not in general position (i.e., if codim $H_{1} \cap \cdots \cap H_{\ell} \neq \ell$ ).

(ii) $\left(H_{\sigma(1)}, \ldots, H_{\sigma(\ell)}\right)=(-1)^{|\sigma|}\left(H_{1}, \ldots, H_{\ell}\right)$ for every permutation $\sigma \in \Sigma_{\ell}$.

(iii) For any $\ell+1$ hyperplanes $H_{1}, \ldots, H_{\ell+1}$ that have a non-empty intersection, $H_{1} \cap \cdots \cap H_{\ell+1} \neq \varnothing$, and that are not in general position:

$$
\sum_{i=1}^{\ell+1}(-1)^{i}\left(H_{1}, \ldots, \widehat{H}_{i}, \ldots, H_{\ell+1}\right)=0
$$

where $\widehat{H}_{i}$ denotes omission.

The abelian group $\mathcal{A}^{\ell}(\mathcal{C}, \mathbb{Z})$ is a free abelian group, (see [6]; [5], Theorem 2.9.2).

The Orlik-Solomon algebra of the arrangement $\mathcal{C}$ is the direct sum $\mathcal{A}^{\bullet}(\mathcal{C}, \mathbb{Z})=\oplus_{\ell=0}^{k} \mathcal{A}^{\ell}(\mathcal{C}, \mathbb{Z})$ endowed with the product given by $\left(H_{1}, \ldots, H_{i}\right) \wedge\left(H_{1}^{\prime}, \ldots, H_{j}^{\prime}\right)=\left(H_{1}, \ldots, H_{i}, H_{1}^{\prime}, \ldots, H_{j}^{\prime}\right)$. It is a graded skew-commutative algebra over $\mathbb{Z}$.

\subsection{Orlik-Solomon Algebra as an Algebra of Differential Forms}

For each hyperplane $H \in \mathcal{C}$, pick a polynomial $f_{H}$ of degree 1 on $\mathbb{C}^{k}$ whose zero set is $H$; that is, let $f_{H}=0$ be an affine equation for $H$. Consider the logarithmic differential form:

$$
\iota(H):=d \log f_{H}=\frac{d f_{H}}{f_{H}}
$$

on $\mathbb{C}^{k}$. We note that $\iota(H)$ does not depend on the choice of $f_{H}$ but only on $H$. Let $\overline{\mathcal{A}} \bullet(\mathcal{C}, \mathbb{Z})$ be the $\mathbb{Z}$-algebra of differential forms generated by 1 and $\iota(H), H \in \mathbb{C}$. The assignment $H \mapsto \iota(H)$ defines an isomorphism $\mathcal{A} \bullet(\mathcal{C}, \mathbb{Z}) \stackrel{\sim}{\rightarrow} \overline{\mathcal{A}} \bullet(\mathcal{C}, \mathbb{Z})$ of graded algebras. Henceforth we shall not distinguish between $\mathcal{A}$ and $\overline{\mathcal{A}}$.

\subsection{Duality—See [5] (cf. [7], Section 2.5)}

The vector spaces $\mathcal{A}^{\ell}(\mathcal{C}, \mathbb{Z})$ and $\mathcal{F}^{\ell}(\mathcal{C}, \mathbb{Z})$ are dual. The pairing $\mathcal{A}^{\ell}(\mathcal{C}, \mathbb{Z}) \otimes \mathcal{F}^{\ell}(\mathcal{C}, \mathbb{Z}) \rightarrow \mathbb{Z}$ is defined as follows. For $H_{j_{1}}, \ldots, H_{j_{\ell}}$ in general position, set $F\left(H_{j_{1}}, \ldots, H_{j_{\ell}}\right)=\left(\tilde{L}^{0} \supset \cdots \supset \tilde{L}^{\ell}\right) \in \mathcal{F}^{\ell}(\mathcal{C}, \mathbb{Z})$, where $\tilde{L}^{0}=\mathbb{C}^{k}$ and:

$$
\tilde{L}^{i}=H_{j_{1}} \cap \cdots \cap H_{j_{i}}, \quad i=1, \ldots, \ell
$$

For any $F=\left(\begin{array}{lllll}L^{0} & \supset & \cdots & \supset & L^{\ell}\end{array}\right) \in \mathcal{F}^{\ell}(\mathcal{C}, \mathbb{Z})$, define $\left\langle\left(H_{j_{1}}, \ldots, H_{j_{\ell}}\right), F\right\rangle=(-1)^{|\sigma|}$, if $F=F\left(H_{j_{\sigma(1)}}, \ldots, H_{j_{\sigma(\ell)}}\right)$ for some $\sigma \in S_{\ell}$, and $\left\langle\left(H_{j_{1}}, \ldots, H_{j_{\ell}}\right), F\right\rangle=0$ otherwise.

\subsection{Flag and Orlik-Solomon Spaces over a Field $\mathbb{F}$}

For any field $\mathbb{F}$ and $\ell=0, \ldots, k$, we define:

$$
\mathcal{F}^{\ell}(\mathcal{C}, \mathbb{F})=\mathcal{F}^{\ell}(\mathcal{C}, \mathbb{Z}) \otimes_{\mathbb{Z}} \mathbb{F}, \quad \mathcal{A}^{\ell}(\mathcal{C}, \mathbb{F})=\mathcal{A}^{\ell}(\mathcal{C}, \mathbb{Z}) \otimes_{\mathbb{Z}} \mathbb{F}
$$

\subsection{Weights}

An arrangement $\mathcal{C}$ is weighted if a map $a: J \rightarrow \mathbb{C}^{\times}, j \mapsto a_{j}$, is given; $a_{j}$ is called the weight of $H_{j}$. For an edge $X_{\alpha}$, define its weight as $a_{\alpha}=\sum_{j \in J_{\alpha}} a_{j}$. 


\subsection{Contravariant Form and Map-See [5]}

The weights determine a symmetric bilinear form $S^{(a)}$ on $\mathcal{F}^{\ell}(\mathcal{C}, \mathbb{C})$, given by:

$$
S^{(a)}\left(F_{1}, F_{2}\right)=\sum_{\left\{j_{1}, \ldots, j_{p}\right\} \subset J} a_{j_{1}} \cdots a_{j_{\ell}}\left\langle\left(H_{j_{1}}, \ldots, H_{j_{\ell}}\right), F_{1}\right\rangle\left\langle\left(H_{j_{1}}, \ldots, H_{j_{\ell}}\right), F_{2}\right\rangle
$$

where the sum is over all unordered $\ell$-element subsets. The form is called the contravariant form. It defines a homomorphism:

$$
\mathcal{S}^{(a)}: \mathcal{F}^{\ell}(\mathcal{C}, \mathbb{C}) \rightarrow \mathcal{F}^{\ell}(\mathcal{C}, \mathbb{C})^{*} \simeq \mathcal{A}^{\ell}(\mathcal{C}, \mathbb{C}), \quad\left(L^{0} \supset \cdots \supset L^{\ell}\right) \mapsto \sum a_{j_{1}} \cdots a_{j_{\ell}}\left(H_{j_{1}}, \ldots, H_{j_{\ell}}\right)
$$

where the sum is taken over all $\ell$-tuples $\left(H_{j_{1}}, \ldots, H_{j_{\ell}}\right)$, such that:

$$
H_{j_{1}} \supset L^{1}, \ldots, H_{j_{\ell}} \supset L^{\ell}
$$

Theorem 1 ([5], Theorem 3.7). For $\ell=1, \ldots, k$, choose a basis of the free abelian group $\mathcal{F}^{\ell}(\mathcal{C}, \mathbb{Z})$. Then with respect to that basis, the determinant of the contravariant form $S^{(a)}$ on $\mathcal{F}^{\ell}(\mathcal{C}, \mathbb{Z})$ equals the product of suitable non-negative integer powers of the weights of all dense edges of $\mathcal{C}$ of codimension not greater than $\ell$.

Corollary 1. If the weights of all dense edges of $\mathcal{C}$ are nonzero, then the contravariant map $\mathcal{S}^{(a)}: \mathcal{F}^{\ell}(\mathcal{C}, \mathbb{C}) \rightarrow$ $\mathcal{A}^{\ell}(\mathcal{C}, \mathbb{C})$ is an isomorphism for all $\ell$.

\subsection{Aomoto Complex}

Define:

$$
v(a)=\sum_{j \in J} a_{j}\left(H_{j}\right) \in \mathcal{A}^{1}(\mathcal{C}, \mathbb{C})
$$

Multiplication by $v(a)$ defines a differential:

$$
d^{(a)}: \mathcal{A}^{\ell}(\mathcal{C}, \mathbb{C}) \rightarrow \mathcal{A}^{\ell+1}(\mathcal{C}, \mathbb{C}), \quad x \mapsto x \wedge v(a)
$$

on $\mathcal{A}^{\bullet}(\mathcal{C}, \mathbb{C}), \quad\left(d^{(a)}\right)^{2}=0$. The complex $\left(\mathcal{A} \bullet(\mathcal{C}, \mathbb{C}), d^{(a)}\right)$ is called the Aomoto complex. The master function corresponding to the weighted arrangement $(\mathcal{C}, a)$ is the function:

$$
\Phi=\Phi_{\mathcal{C}, a}=\prod_{j \in J} f_{H_{j}}^{a_{j}}
$$

where each $f_{H_{j}}=0$ is an affine equation for the hyperplane $H_{j}$. Then $v(a)=d \Phi / \Phi$.

Theorem 2 ([5], Lemma 3.2.5; and [8], Lemma 5.1). The Shapovalov map is a homomorphism of complexes:

$$
\mathcal{S}^{(a)}:\left(\mathcal{F}^{\bullet}(\mathcal{C}, \mathbb{Z}), d\right) \rightarrow\left(\mathcal{A}^{\bullet}(\mathcal{C}, \mathbb{Z}), d^{(a)}\right)
$$

\subsection{Singular Vectors}

An element $v \in \mathcal{F}^{k}(\mathcal{C}, \mathbb{C})$ is called singular if $\left\langle d^{(a)} \mathcal{A}^{\ell-1}(\mathcal{C}, \mathbb{C}), v\right\rangle=0$. Denote by:

$$
\operatorname{Sing} \mathcal{F}^{k}(\mathcal{C}, \mathbb{C}) \subset \mathcal{F}^{k}(\mathcal{C}, \mathbb{C})
$$

the subspace of all singular vectors.

\subsection{Arrangements with Normal Crossings Only}

An essential arrangement $\mathcal{C}$ is with normal crossings only, if exactly $k$ hyperplanes meet at every vertex of $\mathcal{C}$. Assume that $\mathcal{C}$ is an essential arrangement with normal crossings only.

A subset $\left\{j_{1}, \ldots, j_{\ell}\right\} \subset J$ is called independent if the hyperplanes $H_{j_{1}}, \ldots, H_{j_{\ell}}$ intersect transversally. A basis of $\mathcal{A}^{\ell}(\mathcal{C}, \mathbb{C})$ is formed by $\left(H_{j_{1}}, \ldots, H_{j_{\ell}}\right)$, where $\left\{j_{1}<\cdots<j_{\ell}\right\}$ are independent $\ell$-element 
subsets of $J$. The dual basis of $\mathcal{F}^{\ell}(\mathcal{C}, \mathbb{C})$ is formed by the corresponding vectors $F\left(H_{j_{1}}, \ldots, H_{j_{\ell}}\right)$. These bases of $\mathcal{A}^{\ell}(\mathcal{C}, \mathbb{C})$ and $\mathcal{F}^{\ell}(\mathcal{C}, \mathbb{C})$ are called standard.

In $\mathcal{F}^{\ell}(\mathcal{C}, \mathbb{C})$, we have:

$$
F\left(H_{j_{1}}, \ldots, H_{j_{\ell}}\right)=(-1)^{|\sigma|} F\left(H_{j_{\sigma(1)}}, \ldots, H_{j_{\sigma(\ell)}}\right)
$$

for any permutation $\sigma \in S_{\ell}$. For an independent subset $\left\{j_{1}, \ldots, j_{\ell}\right\}$, we have $S^{(a)}\left(F\left(H_{j_{1}}, \ldots, H_{j_{\ell}}\right), F\left(H_{j_{1}}, \ldots, H_{j_{\ell}}\right)\right)=a_{j_{1}} \cdots a_{j_{\ell}}$ and $S^{(a)}\left(F\left(H_{j_{1}}, \ldots, H_{j_{\ell}}\right), F\left(H_{i_{1}}, \ldots, H_{i_{\ell}}\right)\right)=0$ for any distinct elements of the standard basis.

\section{A Family of Parallelly Transported Hyperplanes}

\subsection{An Arrangement in $\mathbb{C}^{n} \times \mathbb{C}^{k}$}

Recall that $J=\{1, \ldots, n\}$. Consider $\mathbb{C}^{k}$ with coordinates $t_{1}, \ldots, t_{k}, \mathbb{C}^{n}$ with coordinates $z_{1}, \ldots, z_{n}$, the projection $\mathbb{C}^{n} \times \mathbb{C}^{k} \rightarrow \mathbb{C}^{n}$. Fix $n$ nonzero linear functions on $\mathbb{C}^{k}, g_{j}=b_{j}^{1} t_{1}+\cdots+b_{j}^{k} t_{k}, j \in J$, where $b_{j}^{i} \in \mathbb{C}$. Define $n$ linear functions on $\mathbb{C}^{n} \times \mathbb{C}^{k}$ :

$$
f_{j}=z_{j}+g_{j}=z_{j}+b_{j}^{1} t_{1}+\cdots+b_{j}^{k} t_{k}, \quad j \in J
$$

In $\mathbb{C}^{n} \times \mathbb{C}^{k}$, define the arrangement:

$$
\tilde{\mathcal{C}}=\left\{\tilde{H}_{j} \mid f_{j}=0, j \in J\right\}
$$

Denote $\tilde{U}=\mathbb{C}^{n} \times \mathbb{C}^{k}-\cup_{j \in J} \tilde{H}_{j}$.

For every fixed $z^{0}=\left(z_{1}^{0}, \ldots, z_{n}^{0}\right)$, the arrangement $\tilde{\mathcal{C}}$ induces an arrangement $\mathcal{C}\left(z^{0}\right)$ in the fiber over $z^{0}$ of the projection. We identify every fiber with $\mathbb{C}^{k}$. Then $\mathcal{C}\left(z^{0}\right)$ consists of hyperplanes $H_{j}\left(z^{0}\right), j \in J$, defined in $\mathbb{C}^{k}$ by the same equations, $f_{j}=0$. Denote:

$$
U\left(\mathcal{C}\left(z^{0}\right)\right)=\mathbb{C}^{k}-\cup_{j \in J} H_{j}\left(z^{0}\right)
$$

as the complement to the arrangement $\mathcal{C}\left(z^{0}\right)$.

We assume that for any $z^{0}$, the arrangement $\mathcal{C}\left(z^{0}\right)$ has a vertex. This means that the span of $\left(g_{j}\right)_{j \in J}$ is $k$-dimensional.

A point $z^{0} \in \mathbb{C}^{n}$ is called good if $\mathcal{C}\left(z^{0}\right)$ has normal crossings only. Good points form the complement in $\mathbb{C}^{n}$ to the union of suitable hyperplanes called the discriminant.

\subsection{Discriminant}

The collection $\left(g_{j}\right)_{j \in J}$ induces a matroid structure $\mathcal{M}_{\mathbb{C}}$ on $J$. A subset $C=\left\{i_{1}, \ldots, i_{r}\right\} \subset J$ is a circuit in $\mathcal{M}_{\mathbb{C}}$ if $\left(g_{i}\right)_{i \in C}$ are linearly dependent but any proper subset of $C$ gives linearly independent $g_{i}{ }^{\prime}$ s.

For a circuit $C=\left\{i_{1}, \ldots, i_{r}\right\}$, let $\left(\lambda_{C}^{i}\right)_{i \in C}$ be a nonzero collection of complex numbers such that $\sum_{i \in C} \lambda_{C}^{i} g_{i}=0$. Such a collection is unique up to multiplication by a nonzero number.

For every circuit $C$, we fix such a collection and denote $f_{C}=\sum_{i \in C} \lambda_{C}^{i} z_{i}$. The equation $f_{C}=0$ defines a hyperplane $H_{C}$ in $\mathbb{C}^{n}$. It is convenient to assume that $\lambda_{C}^{i}=0$ for $i \in J-C$ and write $f_{C}=\sum_{i \in J} \lambda_{C}^{i} z_{i}$.

For any $z^{0} \in \mathbb{C}^{n}$, the hyperplanes $\left(H_{i}\left(z^{0}\right)\right)_{i \in C}$ in $\mathbb{C}^{k}$ have a nonempty intersection if and only if $z^{0} \in H_{C}$. If $z^{0} \in H_{C}$, then the intersection has codimension $r-1$ in $\mathbb{C}^{k}$.

Denote by $\mathfrak{C}_{\mathbb{C}}$ the set of all circuits in $\mathcal{M}_{\mathbb{C}}$. Denote $\Delta=\cup_{\mathbb{C} \in \mathfrak{C}_{\mathbb{C}}} H_{\mathbb{C}}$. The arrangement $\mathcal{C}\left(z^{0}\right)$ in $\mathbb{C}^{k}$ has normal crossings only, if and only if $z^{0} \in \mathbb{C}^{n}-\Delta$. 


\subsection{Good Fibers}

For any $z^{1}, z^{2} \in \mathbb{C}^{n}-\Delta$, the spaces $\mathcal{F}^{\ell}\left(\mathcal{C}\left(z^{1}\right), \mathbb{C}\right), \mathcal{F}^{\ell}\left(\mathcal{C}\left(z^{2}\right), \mathbb{C}\right)$ are canonically identified. Namely, a vector $F\left(H_{j_{1}}\left(z^{1}\right), \ldots, H_{j_{\ell}}\left(z^{1}\right)\right)$ of the first space is identified with the vector $F\left(H_{j_{1}}\left(z^{2}\right), \ldots, H_{j_{\ell}}\left(z^{2}\right)\right)$ of the second.

Assume that weights $a=\left(a_{j}\right)_{j \in J}$ are given. Then each arrangement $\mathcal{C}(z)$ is weighted. The identification of spaces $\mathcal{F}^{\ell}\left(\mathcal{C}\left(z^{1}\right), \mathbb{C}\right), \mathcal{F}^{\ell}\left(\mathcal{C}\left(z^{2}\right), \mathbb{C}\right)$ for $z^{1}, z^{2} \in \mathbb{C}^{n}-\Delta$ identifies the corresponding subspaces $\operatorname{Sing} \mathcal{F}^{k}\left(\mathcal{C}\left(z^{1}\right), \mathbb{C}\right)$ and $\operatorname{Sing} \mathcal{F}^{k}\left(\mathcal{C}\left(z^{2}\right), \mathbb{C}\right)$ and the corresponding contravariant forms.

For a point $z^{0} \in \mathbb{C}^{n}-\Delta$, denote $V_{\mathbb{C}}=\mathcal{F}^{k}\left(\mathcal{C}\left(z^{0}\right), \mathbb{C}\right)$, Sing $V_{\mathbb{C}}=\operatorname{Sing} \mathcal{F}^{k}\left(\mathcal{C}\left(z^{0}\right), \mathbb{C}\right)$. The triple $\left(V_{\mathbb{C}}, \operatorname{Sing} V_{\mathbb{C}}, S^{(a)}\right)$ does not depend on $z^{0} \in \mathbb{C}^{n}-\Delta$ under the above identification.

\subsection{Geometric Hamiltonians (cf. $[9,10])$}

For any circuit $C=\left\{i_{1}, \ldots, i_{r}\right\}$, we define a linear operator $L_{\mathbb{C}}: V_{\mathbb{C}} \rightarrow V_{\mathbb{C}}$ in terms of the standard basis of $V_{\mathbb{C}}$ (see Section 2.11).

For $m=1, \ldots, r$, denote $C_{m}=C-\left\{i_{m}\right\}$. Let $\left\{j_{1}<\cdots<j_{k}\right\} \subset J$ be an independent ordered subset and $F\left(H_{j_{1}}, \ldots, H_{j_{k}}\right)$ be the corresponding element of the standard basis. Define $L_{C}: F\left(H_{j_{1}}, \ldots, H_{j_{k}}\right) \mapsto 0$ if $\left|\left\{j_{1}, \ldots, j_{k}\right\} \cap C\right|<r-1$. If $\left\{j_{1}, \ldots, j_{k}\right\} \cap C=C_{m}$ for some $1 \leqslant m \leqslant r$, then using the skew-symmetry property of Equation (5), we can write:

$$
F\left(H_{j_{1}}, \ldots, H_{j_{k}}\right)= \pm F\left(H_{i_{1}}, H_{i_{2}}, \ldots, \widehat{H_{i_{m}}}, \ldots, H_{i_{r-1}} H_{i_{r}}, H_{s_{1}}, \ldots, H_{s_{k-r+1}}\right)
$$

with $\left\{s_{1}, \ldots, s_{k-r+1}\right\}=\left\{j_{1}, \ldots, j_{k}\right\}-C_{m}$. Define:

$$
\begin{aligned}
& L_{C}: \quad F\left(H_{i_{1}}, \ldots, \widehat{H_{i_{m}}}, \ldots, H_{i_{r}}, H_{s_{1}}, \ldots, H_{s_{k-r+1}}\right) \mapsto \\
&(-1)^{m} \sum_{l=1}^{r}(-1)^{l} a_{i_{l}} F\left(H_{i_{1}}, \ldots, \widehat{H_{i_{l}}}, \ldots, H_{i_{r}}, H_{s_{1}}, \ldots, H_{s_{k-r+1}}\right)
\end{aligned}
$$

Lemma 1 ([9]). The operator $L_{C}$ is symmetric with respect to the contravariant form.

Consider the logarithmic differential one-forms:

$$
\omega_{j}=\frac{d f_{j}}{f_{j}}, j \in J, \quad \omega_{C}=\frac{d f_{C}}{f_{C}}, C \in \mathfrak{C}_{\mathbb{C}}
$$

in variables $t_{1}, \ldots, t_{k}, z_{1}, \ldots, z_{n}$. For any circuit $C=\left\{i_{1}, \ldots, i_{r}\right\}$, we have:

$$
\omega_{i_{1}} \wedge \cdots \wedge \omega_{i_{r}}=\omega_{C} \wedge \sum_{l=1}^{r}(-1)^{l-1} \omega_{i_{1}} \wedge \cdots \wedge \widehat{\omega_{i_{l}}} \wedge \cdots \wedge \omega_{i_{r}}
$$

Lemma 2 ([9], Lemma 4.2; and [10], Lemma 5.4). We have:

$$
\begin{aligned}
& \sum_{\substack{\text { independent } \\
\left\{j_{1}<\cdots<j_{k}\right\} \subset J}}\left(\sum_{j \in J} a_{j} \omega_{j}\right) \wedge \omega_{j_{1}} \wedge \cdots \wedge \omega_{j_{k}} \otimes F\left(H_{j_{1}}, \ldots, H_{j_{k}}\right)= \\
& \sum_{\substack{\text { independent } \\
\left\{j_{1}<\cdots<j_{k}\right\} \subset J}} \sum_{C \in \mathfrak{C}_{\mathbb{C}}} \omega_{C} \wedge \omega_{j_{1}} \wedge \cdots \wedge \omega_{j_{k}} \otimes L_{C} F\left(H_{j_{1}}, \ldots, H_{j_{k}}\right)
\end{aligned}
$$

Proof. The lemma is a direct corollary of the definition of the maps $L_{C}$.

The identity in Equation (8) is called the key identity.

Recall that $\omega_{C}=d f_{C} / f_{C}$ and $f_{C}=\sum_{j \in J} \lambda_{C}^{i} z_{i}$. For $i \in J$, we introduce the $\operatorname{End}_{\mathbb{C}}\left(V_{\mathbb{C}}\right)$-valued rational functions in $z_{1}, \ldots, z_{n}$ by the formula:

$$
K_{i}(z)=\sum_{C \in \mathfrak{C}_{\mathbb{C}}} \frac{\lambda_{C}^{i}}{f_{C}(z)} L_{C}, \quad i \in J
$$


Then:

$$
\sum_{C \in \mathfrak{C}_{\mathbb{C}}} \omega_{C} \otimes L_{C}=\sum_{i \in J} d z_{i} \otimes K_{i}(z)
$$

The functions $K_{i}(z)$ are called geometric Hamiltonians.

Corollary 2. The geometric Hamiltonians are symmetric with respect to the contravariant form, $S^{(a)}\left(K_{i}(z) x, y\right)=S^{(a)}\left(x, K_{i}(z) y\right)$ for $i \in J, x, y \in V_{\mathbb{C}}$.

\subsection{Gauss-Manin Differential Equations}

The Gauss-Manin differential equations with parameter $\kappa \in \mathbb{C}^{\times}$are given by the following

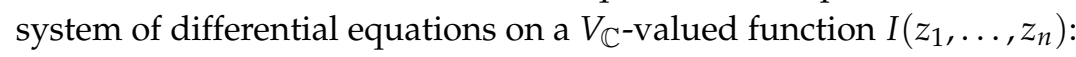

$$
\kappa \frac{\partial I}{\partial z_{i}}(z)=K_{i}(z) I(z), \quad i \in J
$$

where $K_{i}(z)$ are the geometric Hamiltonians defined in Equation (9).

We introduce the master function:

$$
\Phi(z, t, a)=\prod_{j \in J} f_{j}(z, t)^{a_{j}}
$$

on $\tilde{U} \subset \mathbb{C}^{n} \times \mathbb{C}^{k}$. The function $\Phi(z, t, a)^{1 / \kappa}$ defines a rank-one local system $\mathcal{L}_{\kappa}$ on $\tilde{U}$, whose horizontal sections over open subsets of $\tilde{U}$ are univalued branches of $\Phi(z, t, a)^{1 / \kappa}$ multiplied by complex numbers.

For $z^{0} \in \mathbb{C}^{n}-\Delta$ and an element $\gamma\left(z^{0}\right) \in H_{k}\left(U\left(\mathcal{C}\left(z^{0}\right)\right),\left.\mathcal{L}_{\kappa}\right|_{U\left(\mathcal{C}\left(z^{0}\right)\right)}\right)$, we interpret the integration $\operatorname{map} \mathcal{A}^{k}\left(\mathcal{C}\left(z^{0}\right), \mathbb{C}\right)=V^{*} \rightarrow \mathbb{C}, \omega \mapsto \int_{\gamma\left(z^{0}\right)} \Phi\left(z^{0}, t, a\right)^{1 / \kappa} \omega$ as an element of $\mathcal{F}^{k}\left(\mathcal{C}\left(z^{0}\right), \mathbb{C}\right)=V_{\mathbb{C}}$. The vector bundle:

$$
\cup_{z^{0} \in \mathbb{C}^{n}-\Delta} H_{k}\left(U\left(\mathcal{C}\left(z^{0}\right)\right),\left.\mathcal{L}_{\kappa}\right|_{U\left(\mathcal{C}\left(z^{0}\right)\right)}\right) \rightarrow \mathbb{C}^{n}-\Delta
$$

has a canonical flat Gauss-Manin connection. A locally constant section $\gamma: z \mapsto \gamma(z) \in$

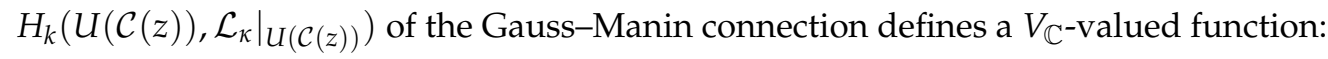

$$
I^{(\gamma)}\left(z_{1}, \ldots, z_{n}\right)=\sum_{\substack{\text { independent } \\\left\{j_{1}<\cdots<j_{k}\right\} \subset J}}\left(\int_{\gamma(z)} \Phi(z, t, a)^{1 / \kappa} \omega_{j_{1}} \wedge \cdots \wedge \omega_{j_{k}}\right) F\left(H_{j_{1}}, \ldots, H_{j_{k}}\right)
$$

The integrals:

$$
I_{j_{1}, \ldots, j_{k}}^{(\gamma)}\left(z_{1}, \ldots, z_{n}\right)=\int_{\gamma(z)} \Phi(z, t, a)^{1 / \kappa} \omega_{j_{1}} \wedge \cdots \wedge \omega_{j_{k}}
$$

are called the multidimensional hypergeometric integrals associated with the master function $\Phi(z, t, a)$.

Theorem 3 ([10]). The function $I^{(\gamma)}$ takes values in $\operatorname{Sing} V_{\mathbb{C}}$ and gives solutions of the Gauss-Manin differential equations.

The condition that the function $I^{(\gamma)}$ takes values in $\operatorname{Sing} V_{\mathbb{C}}$ may be reformulated as the system of equations:

$$
\sum_{j \in J} a_{j} I_{j, j_{2}, \ldots, j_{k}}^{(\gamma)}=0, \quad \text { for } j_{2}, \ldots, j_{k} \in J
$$

\subsection{Proof of Theorem 3}

We sketch the proof following [3,5]. The intermediate statements of this sketch are used further when constructing solutions of the Gauss-Manin differential equations over a finite field $\mathbb{F}_{p}$. The proof of Theorem 3 is based on the following cohomological relations, Equations (21) and (24). 
For any $j_{1}, \ldots, j_{k} \in J$, denote:

$$
d_{j_{1}, \ldots, j_{k}}=\operatorname{det}_{i, l=1}^{k}\left(b_{j_{l}}^{i}\right), \quad W_{j_{1}, \ldots, j_{k}}(z, t)=\frac{d_{j_{1}, \ldots, j_{k}}}{\prod_{l=1}^{k} f_{j_{l}}(z, t)}
$$

We have:

$$
\omega_{j_{1}} \wedge \cdots \wedge \omega_{j_{k}}=W_{j_{1}, \ldots, j_{k}}(z, t) d t_{1} \wedge \cdots \wedge d t_{k}+\ldots
$$

where the dots denote the terms having differentials $d z_{j_{1}}, \ldots, d z_{j_{k}}$. We note that the rational function $W_{j_{1}, \ldots, j_{k}}(z, t)$ has the form:

$$
P_{j_{1}, \ldots, j_{k}}(z, t) \prod_{j \in J} f_{j}(z, t)^{-1}
$$

where $P_{j_{1}, \ldots, j_{k}}(z, t)$ is a polynomial with integer coefficients in variable $z_{1}, \ldots, z_{n}, t_{1}, \ldots, t_{k}$ and $b_{j}^{i}, j \in J$, $i=1, \ldots, n$ (see Equation (6)). For any $j_{2}, \ldots, j_{k} \in J$, we write:

$$
\omega_{j_{2}} \wedge \cdots \wedge \omega_{j_{k}}=\sum_{l=1}^{k} W_{j_{2}, \ldots, j_{k}, l}(z, t) d t_{1} \wedge \cdots \wedge \widehat{d t_{l}} \wedge \cdots \wedge d t_{k}+\ldots
$$

where the dots denote the terms having differentials $d z_{j_{2}}, \ldots, d z_{j_{k}}$, and $W_{j_{1}, \ldots, j_{k} ; l}$ are rational functions in $z, t$ of the form:

$$
P_{j_{1}, \ldots, j_{k} ; l}(z, t) \prod_{j \in J} f_{j}(z, t)^{-1}
$$

Here, $P_{j_{1}, \ldots, j_{k}, l}(z, t)$ are polynomials with integer coefficients in variable $z_{1}, \ldots, z_{n}, t_{1}, \ldots, t_{k}$ and $b_{j}^{i}$, $j \in J, i=1, \ldots, n$ (see Equation (6)). The formula:

$$
v(a) \wedge \omega_{j_{2}} \wedge \cdots \wedge \omega_{j_{k}}=\sum_{j \in J} a_{j} \omega_{j} \wedge \omega_{j_{2}} \wedge \cdots \wedge \omega_{j_{k}}
$$

implies the identity:

$$
\begin{gathered}
\kappa d_{t}\left(\Phi(z, t, a)^{1 / \kappa} \sum_{l=1}^{k} W_{j_{2}, \ldots, j_{k} ; l}(z, t) d t_{1} \wedge \cdots \wedge \widehat{d t_{l}} \wedge \ldots d t_{k}\right) \\
=\sum_{j \in J} a_{j} \Phi(z, t, a)^{1 / \kappa} W_{j, j_{2}, \ldots, j_{k}}(z, t) d t_{1} \wedge \cdots \wedge d t_{k}
\end{gathered}
$$

where $d_{t}$ denotes the differential with respect to the variables $t$.

Now we deduce a corollary of the key identity, Equation (8). Choose $i \in J$. For any independent $\left\{j_{1}<\ldots<j_{k}\right\} \subset J$, we write:

$$
\begin{aligned}
& \Phi(z, t, a)^{1 / \kappa} \omega_{j_{1}} \wedge \cdots \wedge \omega_{j_{k}}=\Phi(z, t, a)^{1 / \kappa} W_{j_{1}, \ldots, j_{k}}(z, t) d t_{1} \wedge \cdots \wedge d t_{k} \\
& \quad+d z_{i} \wedge\left(\Phi(z, t, a)^{1 / \kappa} \sum_{l=1}^{k} W_{j_{1}, \ldots, j_{k} ;, i, l}(z, t) d t_{1} \wedge \cdots \wedge \widehat{d t_{l}} \wedge \cdots \wedge d t_{k}\right)+\ldots
\end{aligned}
$$

where the dots denote the terms that contain $d z_{j}$ with $j \neq i$, and the coefficients $W_{j_{1}, \ldots, j_{k} ; i, l}(z, t)$ are rational functions in $z$, $t$ of the form:

$$
P_{j_{1}, \ldots, j_{k} ; i, l}(t, z) \prod_{j \in J} f_{j}(z, t)^{-1}
$$

Here, $P_{j_{1}, \ldots, j_{k} ; i, l}(z, t)$ are polynomials with integer coefficients in variable $z_{1}, \ldots, z_{n}, t_{1}, \ldots, t_{k}$ and $b_{j^{\prime}}^{i}, j \in J, i=1, \ldots, n$ (see Equation (6)).

Equation (8) implies that for any $i \in J$, we have:

$$
\begin{aligned}
& \kappa \sum_{\substack{\text { independent } \\
\left\{j_{1}<\cdots<j_{k}\right\} \subset J}}\left(\frac{\partial}{\partial z_{i}}\left(\Phi(z, t, a)^{1 / \kappa} W_{j_{1}, \ldots, j_{k}}(z, t)\right) d t_{1} \wedge \cdots \wedge d t_{k}\right. \\
& \left.+d_{t}\left(\Phi(z, t, a)^{1 / \kappa} \sum_{l=1}^{n} W_{j_{1}, \ldots, j_{k} ; i, l}(t, z) d t_{1} \wedge \cdots \wedge \widehat{d t_{l}} \wedge \cdots \wedge d t_{k}\right)\right) F\left(H_{j_{1}}, \ldots, H_{j_{k}}\right) \\
& \quad=K_{i}(z) \sum_{\substack{\text { independent } \\
\left\{j_{1}<\cdots<j_{k}\right\} \subset J}} \Phi(z, t, a)^{1 / \kappa} W_{j_{1}, \ldots, j_{k}}(z, t) d t_{1} \wedge \cdots \wedge d t_{k} F\left(H_{j_{1}}, \ldots, H_{j_{k}}\right)
\end{aligned}
$$


where $d_{t}$ denotes the differential with respect to the variables $t$.

Integrating both sides of Equations (21) and (24) over $\gamma(z)$ and using Stokes' theorem, we obtain Equations (14) and (11) for the vector $I^{(\gamma)}(z)$ in Equation (13). Theorem 3 is proved.

\subsection{Remarks}

It is known from [5] that for generic $\kappa$, all $\operatorname{Sing} V_{\mathbb{C}}$-valued solutions of the Gauss-Manin Equation (11) are given by Equation (13). Hence, we have the following statement.

Theorem 4 ([10]). The geometric Hamiltonians $H_{i}(z), i \in J$ preserve $\operatorname{Sing} V_{\mathbb{C}}$ and commute on $\operatorname{Sing} V_{\mathbb{C}}$, namely, $\left[\left.H_{i}\left(z^{0}\right)\right|_{\text {Sing } V_{\mathbb{C}}},\left.H_{j}\left(z^{0}\right)\right|_{\text {Sing } V_{\mathbb{C}}}\right]=0$ for all $i, j \in J$ and $z^{0} \in \mathbb{C}^{n}-\Delta$.

\section{Reduction Modulo $p$ of a Family of Parallelly Transported Hyperplanes}

\subsection{An Arrangement in $\mathbb{C}^{n} \times \mathbb{C}^{k}$ over $\mathbb{Z}$}

Similarly to Section 3.1 , we consider $\mathbb{C}^{k}$ with coordinates $t_{1}, \ldots, t_{k}, \mathbb{C}^{n}$ with coordinates $z_{1}, \ldots, z_{n}$, the projection $\mathbb{C}^{n} \times \mathbb{C}^{k} \rightarrow \mathbb{C}^{n}$. Fix $n$ nonzero linear functions on $\mathbb{C}^{k}, g_{j}=b_{j}^{1} t_{1}+\cdots+b_{j}^{k} t_{k}, j \in J$, with integer coefficients $b_{j}^{i} \in \mathbb{Z}$. Define $n$ linear functions on $\mathbb{C}^{n} \times \mathbb{C}^{k}$ :

$$
f_{j}=z_{j}+g_{j}=z_{j}+b_{j}^{1} t_{1}+\cdots+b_{j}^{k} t_{k}
$$

where $j \in J$.

Recall the matroid structure $\mathcal{M}_{\mathbb{C}}$ on $J$, the set $\mathfrak{C}_{\mathbb{C}}$ of all circuits in $\mathcal{M}_{\mathbb{C}}$, and the linear functions $f_{C}=\sum_{i \in J} \lambda_{C}^{i} z_{i}$ labeled by $C \in \mathfrak{C}_{\mathbb{C}}$, where the functions are defined in Section 3.2. Each of these functions is determined up to multiplication by a nonzero constant.

Definition 1. We fix the coefficients $\left(\lambda_{C}^{i}\right)_{i \in J}$ to be integers such that the greatest common divisor of $\left(\lambda_{C}^{i}\right)_{i \in J}$ equals 1.

This is possible as all $b_{j}^{i}$ are integers. This choice of the coefficients defines the function $f_{C}$ uniquely up to multiplication by \pm 1 .

Let $p$ be a prime integer and $\mathbb{F}_{p}$ be the field with $p$ elements. Let []$: \mathbb{Z} \rightarrow \mathbb{F}_{p}$ be the natural projection. We introduce the following linear functions in $z, t$ with coefficients in $\mathbb{F}_{p}$ :

$$
\begin{aligned}
{[g]_{j}: } & =\sum_{i=1}^{k}\left[b_{j}^{i}\right] t_{i}, \quad[f]_{j}:=z_{j}+[g]_{j}, \quad j \in J \\
{[f]_{C}: } & =\sum_{i \in J}\left[\lambda_{C}^{i}\right] z_{i}, \quad C \in \mathfrak{C}_{\mathbb{C}}
\end{aligned}
$$

The collection $\left([g]_{j}\right)_{j \in J}$ induces a matroid structure $\mathcal{M}_{\mathbb{F}_{p}}$ on $J$. A subset $C=\left\{i_{1}, \ldots, i_{r}\right\} \subset J$ is a circuit in $\mathcal{M}_{\mathbb{F}_{p}}$ if $\left([g]_{i}\right)_{i \in C}$ are linearly dependent over $\mathbb{F}_{p}$ but any proper subset of $C$ gives linearly independent $[g]_{i}$ 's.

Definition 2. We say that a prime integer $p$ is good with respect to the collection of linear functions $\left(g_{j}\right)_{j \in J}$ if all linear functions in Equation (26) are nonzero and the matroid structures $\mathcal{M}_{\mathbb{C}}$ and $\mathcal{M}_{\mathbb{F}_{p}}$ on J are the same.

In the following, we always assume that $p$ is good with respect to the collection of linear functions $\left(g_{j}\right)_{j \in J}$.

We have logarithmic differential forms:

$$
[\omega]_{j}=\frac{d[f]_{j}}{[f]_{j}}, j \in J, \quad[\omega]_{C}=\frac{d[f]_{C}}{[f]_{C}}, C \in \mathfrak{C}_{\mathbb{F}_{p}}=\mathfrak{C}_{\mathbb{C}}
$$


in variables $t_{1}, \ldots, t_{k}, z_{1}, \ldots, z_{n}$ with coefficients in $\mathbb{F}_{p}$. For any circuit $C=\left\{i_{1}, \ldots, i_{r}\right\}$, we have:

$$
[\omega]_{i_{1}} \wedge \cdots \wedge[\omega]_{i_{r}}=[\omega]_{C} \wedge \sum_{l=1}^{r}(-1)^{l-1}[\omega]_{i_{1}} \wedge \cdots \wedge \widehat{[\omega]_{i_{l}}} \wedge \cdots \wedge[\omega]_{i_{r}}
$$

Assume that the nonzero integer weights $a=\left(a_{j}\right)_{j \in J}$ are given, where $a_{j} \in \mathbb{Z}, a_{j} \neq 0$. The constructions of Section 3 give us the following:

(i) A vector space $V_{\mathbb{F}_{p}}$ over $\mathbb{F}_{p}$ with standard basis $\left(F\left(H_{j_{1}}, \ldots, H_{j_{k}}\right)\right)$ indexed by all independent subsets $\left\{j_{1}<\cdots<j_{k}\right\}$ of $J$.

(ii) A vector subspace $\operatorname{Sing} V_{\mathbb{F}_{p}} \subset V_{\mathbb{F}_{p}}$ consisting of all linear combinations

$\sum_{\substack{\text { independent } \\\left\{j_{1}<\cdots<j_{k}\right\} \subset J}} I_{j_{1}, \ldots, j_{k}} F\left(H_{j_{1}}, \ldots, H_{j_{k}}\right)$ satisfying the equations:

$$
\sum_{j \in J}\left[a_{j}\right] I_{j, j_{2}, \ldots, j_{k}}=0, \quad \text { for } j_{2}, \ldots, j_{k} \in J
$$

(iii) A symmetric bilinear $\mathbb{F}_{p}$-valued contravariant form $[S]^{(a)}$ on $V_{\mathbb{F}_{p}}$ defined by the formulas:

$$
[S]^{(a)}\left(F\left(H_{j_{1}}, \ldots, H_{j_{k}}\right), F\left(H_{j_{1}}, \ldots, H_{j_{k}}\right)\right)=\left[a_{j_{1}}\right] \cdots\left[a_{j_{k}}\right]
$$

for any independent $\left\{j_{1}<\cdots<j_{k}\right\}$ and $[S]^{(a)}\left(F\left(H_{j_{1}}, \ldots, H_{j_{\ell}}\right), F\left(H_{i_{1}}, \ldots, H_{i_{\ell}}\right)\right)=0$ for any distinct elements of the standard basis.

For any circuit $C=\left\{i_{1}, \ldots, i_{r}\right\}$, we define a linear operator $[L]_{C}: V_{\mathbb{F}_{p}} \rightarrow V_{\mathbb{F}_{p}}$ by the formula of Section 3.4, in which the numbers $a_{i_{l}}$ are replaced with $\left[a_{i_{l}}\right]$. We have the key identity:

$$
\begin{aligned}
& \sum_{\substack{\text { independent } \\
\left\{j_{1}<\cdots<j_{k}\right\} \subset J}}\left(\sum_{j \in J}\left[a_{j}\right][\omega]_{j}\right) \wedge[\omega]_{j_{1}} \wedge \cdots \wedge[\omega]_{j_{k}} \otimes F\left(H_{j_{1}}, \ldots, H_{j_{k}}\right)= \\
& \quad \sum_{\substack{\text { independent } \\
\left\{j_{1}<\cdots<j_{k}\right\} \subset J}} \sum_{C \in \mathfrak{C}_{\mathbb{F}_{p}}}[\omega]_{C} \wedge[\omega]_{j_{1}} \wedge \cdots \wedge[\omega]_{j_{k}} \otimes[L]_{C} F\left(H_{j_{1}}, \ldots, H_{j_{k}}\right)
\end{aligned}
$$

For $i \in J$, we define the $\operatorname{End}_{\mathbb{F}_{p}}\left(V_{\mathbb{F}_{p}}\right)$-valued rational functions in $z_{1}, \ldots, z_{n}$ by the formula:

$$
[K]_{i}(z)=\sum_{C \in \mathfrak{C}_{\mathbb{F}_{p}}} \frac{\left[\lambda_{C}^{i}\right]}{[f]_{C}(z)}[L]_{C}, \quad i \in J
$$

We call the functions $[K]_{i}(z)$ the geometric Hamiltonians. The geometric Hamiltonians are symmetric with respect to the contravariant form $\left[S^{(a)}\right]\left([K]_{i}(z) x, y\right)=[S]^{(a)}\left(x,[K]_{i}(z) y\right)$ for $i \in J$, $x, y \in V_{\mathbb{F}_{p}}$.

The Gauss-Manin differential equations over $\mathbb{F}_{p}$ with parameter $[\kappa] \in \mathbb{F}_{p}^{\times}$are given by the following system of differential equations:

$$
[\kappa] \frac{\partial I}{\partial z_{i}}(z)=[K]_{i}(z) I(z), \quad i \in J
$$

The goal of this paper is to construct polynomial $\operatorname{Sing} V_{\mathbb{F}_{p}}$-valued solutions of these differential equations.

\subsection{Polynomial Solutions}

Let a prime integer $p$ be good with respect to $\left(g_{j}\right)_{j \in J}$. Let $a=\left(a_{j}\right)_{j \in J}$ be nonzero integer weights $a_{j} \in \mathbb{Z}, a_{j} \neq 0$.

Choose positive integers $A=\left(A_{1}, \ldots, A_{n}\right)$, such that:

$$
\left[A_{j}\right]=\frac{\left[a_{j}\right]}{[\kappa]}
$$


in $\mathbb{F}_{p}$. Introduce the master polynomial:

$$
\Phi(z, t, A)=\prod_{j \in J} f_{j}(z, t)^{A_{j}} \in \mathbb{Z}\left[z_{1}, \ldots, z_{n}, t_{1}, \ldots, t_{k}\right]
$$

where $f_{j}(z, t)$ are defined in Equation (25). For any $j_{1}, \ldots, j_{k}$, the function $X_{j_{1}, \ldots, j_{k}}(z, t, A):=$ $\Phi(z, t, A) \frac{d_{j_{1}, \ldots, j_{k}}}{\prod_{l=1}^{k} f_{j_{l}}(z, t)}$ is a polynomial in $z, t$ with integer coefficients. For fixed $q=\left(q_{1}, \ldots, q_{k}\right) \in \mathbb{Z}^{k}$, consider the Taylor expansion:

$$
X_{j_{1}, \ldots, j_{k}}(z, t, A)=\sum_{i_{1}, \ldots, i_{k} \geqslant 0} I_{j_{1}, \ldots, j_{k}}^{i_{1}, i_{k}}(z, q, A)\left(t_{1}-q_{1}\right)^{i_{1}} \ldots\left(t_{k}-q_{k}\right)^{i_{k}}
$$

where $I_{j_{1}, \ldots, j_{k}}^{i_{1}, \ldots, i_{k}}(z, q, A) \in \mathbb{Z}\left[z_{1}, \ldots, z_{n}\right]$ for any $i_{1}, \ldots, i_{k}$. We denote by $[I]_{j_{1}, \ldots, j_{k}}^{i_{1}, \ldots, i_{k}}(z, q, A)$ the projection of $I_{j_{1}, \ldots, j_{k}}^{i_{1}, \ldots, i_{k}}(z, q, A)$ to $\mathbb{F}_{p}\left[z_{1}, \ldots, z_{n}\right]$. Denote:

$$
\begin{aligned}
& {[I]^{i_{1}, \ldots, i_{k}}(z, q, A)} \\
& =\sum_{\substack{\text { independent } \\
\left\{j_{1}<\cdots<j_{k}\right\} \subset J}}[I]_{j_{1}, \ldots, j_{k}}^{i_{1}, \ldots, i_{k}}(z, q, A) F\left(H_{j_{1}}, \ldots, H_{j_{k}}\right) \quad \in \quad V_{\mathbb{F}_{p}} \otimes \mathbb{F}_{p}\left[z_{1}, \ldots, z_{n}\right]
\end{aligned}
$$

Theorem 5. Let a prime integer $p$ be good with respect to $\left(g_{j}\right)_{j \in J}$. Then for any integers $A=\left(A_{1}, \ldots, A_{n}\right)$ satisfying Equation (31), any integers $q=\left(q_{1}, \ldots, q_{k}\right)$, and any positive integers $1=\left(l_{1}, \ldots, l_{k}\right)$, the polynomial function $I(z)=[I]^{l_{1} p-1, \ldots, l_{k} p-1}(z, q, A)$ satisfies the algebraic equations in Equation (27) and the Gauss-Manin differential equations in Equation (30).

The parameters $A, q, l_{1} p-1, \ldots, l_{k} p-1$ of the solution $I(z)$ are analogs of the locally constant cycles $\gamma(z)$ in Section 3.5.

We note that the space of polynomial solutions of Equations (27) and (30) is a module over the $\operatorname{ring} \mathbb{F}_{p}\left[z_{1}^{p}, \ldots, z_{n}^{p}\right]$, as $\frac{\partial z_{i}^{p}}{\partial z_{j}}=0$.

Proof. To prove that $I(z)$ satisfies Equations (27) and (30), consider the Taylor expansions at $t=q$ of both sides of Equations (21) and (24) divided by $d t_{1} \wedge \cdots \wedge d t_{k}$. We note that the Taylor expansions are well defined as a result of Equations (17), (19) and (23). We project the Taylor expansions to $V_{\mathbb{F}_{p}} \otimes \mathbb{F}_{p}\left[z_{1}, \ldots, z_{n}\right]$. Then the terms coming from the $d_{t}$-summands equal zero, as $d\left(t_{i}^{l_{i} p}\right) / d t_{i}=$ $l_{i} p t_{i}^{l_{i} p-1} \equiv 0(\bmod p)$.

\subsection{Relation of Solutions to Integrals over $\mathbb{F}_{p}^{k}$}

For a polynomial $F\left(t_{1}, \ldots, t_{k}\right) \in \mathbb{F}_{p}\left[t_{1}, \ldots, t_{k}\right]$ and a subset $\gamma \subset \mathbb{F}_{p}^{k}$, we define the integral:

$$
\int_{\gamma} F\left(t_{1}, \ldots, t_{k}\right) d t_{1} \wedge \cdots \wedge d t_{k}:=\sum_{\left(t_{1}, \ldots, t_{k}\right) \in \gamma} F\left(t_{1}, \ldots, t_{k}\right)
$$

We consider the vector of polynomials:

$$
\begin{aligned}
& F(x, t, A) \\
& =\sum_{\substack{\text { independent } \\
\left\{j_{1}<\cdots<j_{k}\right\} \subset J}} \prod_{j \in J}\left([f]_{j}(x, t)\right)^{A_{j}} \frac{\left[d_{j_{1}, \ldots, j_{k}}\right]}{\prod_{l=1}^{k}[f]_{j_{l}}(x, t)} F\left(H_{j_{1}}, \ldots, H_{j_{k}}\right) \in V_{\mathbb{F}_{p}} \otimes \mathbb{F}_{p}\left[t_{1}, \ldots, t_{k}\right]
\end{aligned}
$$

Theorem 6. Let $x=\left(x_{1}, \ldots, x_{n}\right) \in \mathbb{F}_{p}$. Let $[I]^{p-1, \ldots, p-1}(z, q, A)$ be the solution of Equations (27) and (30) considered in Theorem 5 for $\left(l_{1}, \ldots, l_{k}\right)=(1, \ldots, 1)$. 
(i) If $\operatorname{deg}_{t_{i}} F(x, t, A)<2 p-2$ for $i=1, \ldots, k$, then:

$$
[I]^{(p-1, \ldots, p-1)}(x, q, A)=(-1)^{k} \int_{\mathbb{F}_{p}^{k}} F(x, t, A) d t_{1} \wedge \cdots \wedge d t_{k}
$$

(ii) If the integers $A=\left(A_{j}\right)_{j \in J}$ are such that:

$$
A_{1}+\cdots+A_{n}-k<(k+1)(p-1)
$$

then Equation (36) holds.

Proof. Part 1 follows from the statement that for a positive integer $i$,

$$
\sum_{t \in \mathbb{F}_{p}} t^{i} \text { equals }-1 \text { if }(p-1) \mid i \text { and equals zero otherwise }
$$

Part 2 also follows from Equation (38) by the following reason. The polynomial $P=\prod_{j \in J}\left([f]_{j}(x, t)\right)^{A_{j}} \frac{\left[d_{j_{1}, \ldots, j_{k}}\right]}{\prod_{l=1}^{k}[f]_{j_{l}}(x, t)}$ is a product of $A_{1}+\cdots+A_{n}-k$ linear functions in $t$. If Equation (37) holds, then $\left(t_{1} \ldots t_{k}\right)^{p-1}$ is the only monomial $t_{1}^{m_{1}} \ldots t_{k}^{m_{k}}$ of the Taylor expansion of that polynomial, such that $m_{l}>0$ and $(p-1) \mid m_{l}$ for $l=1, \ldots, k$.

The integral in Equation (36) is a $p$-analog of the hypergeometric integral of Equation (13) (see also Section 5).

\section{Examples}

\subsection{Case $k=1-$ See [3]}

Let $\kappa, a=\left(a_{1}, \ldots, a_{n}\right)$ be nonzero complex numbers. We consider the master function of complex variables:

$$
\Phi\left(t_{1}, z_{1}, \ldots, z_{n}, a\right)=\prod_{i=1}^{n}\left(t_{1}+z_{i}\right)^{a_{i}}
$$

Let $z^{0}=\left(z_{1}^{0}, \ldots, z_{n}^{0}\right) \in \mathbb{C}^{n}$ be a vector with distinct coordinates. We consider the $n$-vector $I^{(\gamma)}\left(z^{0}\right)=\left(I_{1}\left(z^{0}\right), \ldots, I_{n}\left(z^{0}\right)\right)$, where:

$$
I_{j}=\int_{\gamma\left(z^{0}\right)} \Phi\left(t_{1}, z_{1}^{0}, \ldots, z_{n}^{0}, a\right)^{1 / \kappa} \frac{d t_{1}}{t_{1}+z_{j}^{0}}, \quad j=1, \ldots, n
$$

The integrals are over a closed (Pochhammer) curve $\gamma\left(z^{0}\right)$ in $\mathbb{C}-\left\{z_{1}^{0}, \ldots, z_{n}^{0}\right\}$ on which one fixes a uni-valued branch of the master function to make the integral well-defined. Starting from such a curve chosen for a given $\left\{z_{1}^{0}, \ldots, z_{n}^{n}\right\} \subset \mathbb{C}$, the vector $I^{(\gamma)}\left(z^{0}\right)$ can be analytically continued as a multivalued holomorphic function of $z$ to the complement in $\mathbb{C}^{n}$ to the union of the diagonal hyperplanes $z_{i}=z_{j}$.

Theorem 7. The vector $I^{(\gamma)}(z)$ satisfies the algebraic equation:

$$
a_{1} I_{1}(z)+\cdots+a_{n} I_{n}(z)=0
$$

and the differential equations:

$$
\kappa \frac{\partial I}{\partial z_{i}}=\sum_{j \neq i} \frac{\Omega_{i, j}}{z_{i}-z_{j}} I, \quad i=1, \ldots, n
$$


where:

$$
\Omega_{i, j}=\left(\begin{array}{ccccc} 
& i & & j & \\
& \vdots & & \vdots & \\
i \cdots & -a_{j} & \cdots & a_{j} & \cdots \\
& \vdots & & \vdots & \\
j \cdots & a_{i} & \cdots & -a_{i} & \cdots \\
& \vdots & & \vdots &
\end{array}\right)
$$

and all the remaining entries equal zero, (see [5]; [11], Section 1.1).

Example 1. Let $\kappa=2, n=3$, and $a_{1}=a_{2}=a_{3}=-1$. Then $I^{(\gamma)}(z)=\left(I_{1}(z), I_{2}(z), I_{3}(z)\right)$, where:

$$
I_{j}(z)=\int_{\gamma(z)} \frac{1}{\sqrt{\left(t+z_{1}\right)\left(t+z_{2}\right)\left(t+z_{3}\right)}} \frac{d t}{t+z_{j}}
$$

In this case, the curve $\gamma(z)$ may be thought of as a closed path on the elliptic curve:

$$
y^{2}=\left(t+z_{1}\right)\left(t+z_{2}\right)\left(t+z_{3}\right)
$$

Each of these integrals is an elliptic integral. Such an integral is a branch of an analytic continuation of a suitable Euler hypergeometric function up to a change of variables.

Example 2. Let $p>3$ be a prime integer. Let $\kappa=2$, and $a_{1}=\cdots=a_{n}=-1$ (cf. Example 1). For such $\kappa$ and $a_{j}$, the algebraic Equation (41) and the differential Equation (42) are well-defined when reduced modulo $p$. Choose the master polynomial:

$$
\Phi\left(t_{1}, z_{1}, \ldots, z_{n}\right)=\prod_{i=1}^{n}\left(t_{1}+z_{i}\right)^{\frac{p-1}{2}}
$$

Consider the Taylor expansion of the polynomial (see (35)):

$$
F\left(t_{1}, z\right)=\prod_{i=1}^{n}\left(t_{1}+z_{i}\right)^{\frac{p-1}{2}}\left(\frac{1}{t_{1}+z_{1}}, \ldots, \frac{1}{t_{1}+z_{n}}\right)=\sum_{i} I^{i}(z) t_{1}^{i}
$$

Let $[I]^{i}(z)$ be the projection of $I^{i}(z)$ to $\left(\mathbb{F}_{p}[z]\right)^{n}$. Then the vector $I(z):=[I]^{p-1}(z)$ is a solution of the differential Equation (42) over $\mathbb{F}_{p}[z]$ and $I_{1}(z)+\cdots+I_{n}(z)=0$ (see Theorem 5).

If $n \leqslant 4$ and $x=\left(x_{1}, \ldots, x_{n}\right) \in \mathbb{F}_{p}^{n}$, then:

$$
I(x)=\int_{\mathbb{F}_{p}} F\left(t_{1}, x\right) d t_{1}
$$

by Theorem 6 .

Let $x=\left(x_{1}, \ldots, x_{n}\right) \in \mathbb{F}_{p}^{k}$. Let $\Gamma(x)$ be the affine curve:

$$
y^{2}=\left(t_{1}+x_{1}\right) \ldots\left(t_{1}+x_{n}\right)
$$

over $\mathbb{F}_{p}$. For a rational function $h: \Gamma(x) \rightarrow \mathbb{F}_{p}$, define the integral:

$$
\int_{\Gamma(x)} h=\sum_{P \in \Gamma(x)}^{\prime} h(P)
$$

as the sum over all points $P \in \Gamma(x)$, where $h(P)$ is defined. 
Theorem 8. Let $n$ equal 3 or 4 . Let $[I]^{p-1}(x)=\left([I]_{1}^{p-1}(x), \ldots,[I]_{n}^{p-1}(x)\right)$ be the vector of polynomials obtained from Equation (45). Then:

$$
\int_{\Gamma(x)} \frac{1}{t_{1}+x_{j}}=-[I]_{j}^{p-1}(x), \quad j=1, \ldots, n
$$

Remark 1. Theorems 5 and 8 say that the integrals $\int_{\Gamma\left(x_{1}, \ldots, x_{n}\right)} \frac{1}{t_{1}+x_{j}}$ are polynomials in $x_{1}, \ldots, x_{n} \in \mathbb{F}_{p}$ and the tuple of polynomials:

$$
\left(\int_{\Gamma\left(x_{1}, \ldots, x_{n}\right)} \frac{1}{t_{1}+x_{1}}, \ldots, \int_{\Gamma\left(x_{1}, \ldots, x_{n}\right)} \frac{1}{t_{1}+x_{n}}\right)
$$

in these discrete variables satisfies the system of Gauss-Manin differential equations (cf. Example 1).

Remark 2. In [1], Section 2 and in [2], an equation analogous to Equation (48) for $n=3$ is considered, where the left-hand side is the number of points on $\Gamma\left(x_{1}, x_{2}, x_{3}\right)$ over $\mathbb{F}_{p}$ and the right-hand side is the reduction modulo $p$ of a solution of a second-order Gauss hypergeometric differential equation. We note that the number of points on $\Gamma\left(x_{1}, x_{2}, x_{3}\right)$ is the discrete integral over $\Gamma\left(x_{1}, x_{2}, x_{3}\right)$ of the constant function $h=1$.

Proof of Theorem 8. The proof is analogous to the reasoning in [1], Section 2 and [2]. It is easy to see that:

$$
\begin{aligned}
& \int_{\Gamma\left(x_{1}, \ldots, x_{n}\right)} \frac{1}{t_{1}+x_{j}}=\sum_{t_{1} \in \mathbb{F}_{p}, t_{1} \neq x_{j}} \frac{1}{t_{1}+x_{j}}+\sum_{t_{1} \in \mathbb{F}_{p}} \frac{1}{t_{1}+x_{j}} \prod_{s=1}^{n}\left(t_{1}+x_{s}\right)^{\frac{p-1}{2}} \\
& =\sum_{t_{1} \in \mathbb{F}_{p}}\left(t_{1}+x_{j}\right)^{p-2}+\sum_{t_{1} \in \mathbb{F}_{p}} \sum_{i}[I]_{j}^{i}\left(x_{1}, \ldots, x_{n}\right) t_{1}^{i}=-[I]_{j}^{p-1}\left(x_{1}, \ldots, x_{n}\right)
\end{aligned}
$$

We note that $\sum_{t_{1} \in \mathbb{F}_{p}} \sum_{i}[I]_{j}^{i}\left(x_{1}, \ldots, x_{n}\right) t_{1}^{i}=-[I]_{j}^{p-1}\left(x_{1}, \ldots, x_{n}\right)$, as for $n=3,4$, the degree of the left-hand side is less than $2 p-2$ (see Theorem 6).

See more examples with $k=1$ in ([3], Section 1$)$.

\subsection{Counting on Two-Folded Covers}

As in Section 4.1, we consider $n$ nonzero linear functions on $\mathbb{C}^{k}, g_{j}=b_{j}^{1} t_{1}+\cdots+b_{j}^{k} t_{k}, j \in J$, with integer coefficients $b_{j}^{i} \in \mathbb{Z}$. Let a prime integer $p>3$ be good with respect to $\left(g_{j}\right)_{j \in J}$.

Assume that all weights $\left(a_{j}\right)_{j \in J}$ are equal to -1 and $\kappa=2$. Under these assumptions, consider the algebraic Equation (27) and differential Equation (30). To construct a solution of these equations, choose a master polynomial $\Phi(z, t)=\prod_{j \in J} f_{j}(z, t)^{\frac{p-1}{2}}$, and consider the Taylor expansion of the polynomial:

$$
F(z, t)=\sum_{\substack{\text { independent } \\\left\{j_{1}<\cdots<j_{k}\right\} \subset j}} \Phi(z, t) \frac{d_{j_{1}, \ldots, j_{k}}}{f_{j_{1}}(z, t) \ldots f_{j_{k}}(z, t)} F\left(H_{j_{1}}, \ldots, H_{j_{k}}\right)
$$

at $t=0$, to obtain the solution:

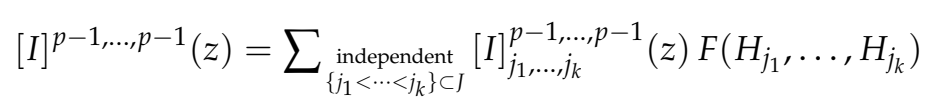

of the algebraic Equation (27) and differential Equation (30) by taking the coefficient of $\left(t_{1} \ldots t_{k}\right)^{p-1}$ of the Taylor expansion (see Theorem 5).

Let $x=\left(x_{1}, \ldots, x_{n}\right) \in \mathbb{F}_{p}^{n}$. Let $\Gamma(x)$ be the affine hypersurface:

$$
y^{2}=\prod_{j \in J}[f]_{j}(x, t)
$$

over $\mathbb{F}_{p}$. Recall that $[f]_{j}(x, t)=\left[b_{j}^{1}\right] t_{1}+\cdots+\left[b_{j}^{k}\right] t_{k}+x_{j}$, where []$: \mathbb{Z} \rightarrow \mathbb{F}_{p}$ is the natural projection. 
For a rational function $h: \Gamma(x) \rightarrow \mathbb{F}_{p}$ define the integral:

$$
\int_{\Gamma(x)} h=\sum_{P \in \Gamma(x)}^{\prime} h(P)
$$

as the sum over all points $P \in \Gamma(x)$ with well-defined $h(P)$.

Theorem 9. Let:

$$
n \frac{p-1}{2}-k<(k+1)(p-1)
$$

Let $[I]^{p-1, \ldots, p-1}(x)$ be the vector in Equation (50) at $z=x$. Then for any independent $\left\{j_{1}<\cdots<j_{k}\right\} \subset$ $J$, we have:

$$
\int_{\Gamma(x)} \frac{\left[d_{j_{1}, \ldots, j_{k}}\right]}{\prod_{l=1}^{k}[f]_{j_{l}}(x, t)}=(-1)^{k}[I]_{j_{1}, \ldots, j_{k}}^{p-1, \ldots, p-1}(x)
$$

This theorem is a generalization of Theorem 8 . Theorem 9 states that the integrals in the left-hand side of Equation (48) are polynomials in $x_{1}, \ldots, x_{n} \in \mathbb{F}_{p}$ and satisfy the algebraic Equation (27) and differential Equation (30).

Proof. It is easy to see the following cf. [1,2]:

$$
\begin{aligned}
\int_{\Gamma(x)} & \frac{\left[d_{j_{1}, \ldots, j_{k}}\right]}{\prod_{l=1}^{k}[f]_{j_{l}}(x, t)} \\
& =\sum_{\substack{\Pi_{l=1}^{k}[f]_{j_{l}}^{k}(x, t) \neq 0 \\
\prod_{l=1}^{k}[f]_{j_{l}}(x, t)}} \frac{\left[d_{j_{1}, \ldots, j_{k}}\right]}{\prod_{t \in \mathbb{F}_{p}^{k}}^{k}} \frac{\left[d_{j_{1}, \ldots, j_{k}}\right]}{\prod_{l=1}^{k}[f]_{j_{l}}(x, t)}\left(\prod_{j \in J}[f]_{j}(x, t)\right)^{\frac{p-1}{2}}
\end{aligned}
$$

Lemma 3. The first sum in the right-hand side of Equation (55) equals zero.

Proof. Because $p$ is good and $\left\{j_{1}, \ldots, j_{k}\right\}$ is independent, we have $\left[d_{j_{1}, \ldots, j_{k}}\right] \neq 0$. Hence we may choose $s_{l}:=[f]_{j_{l}}(x, t), l=1, \ldots, k$, to be affine coordinates on $\mathbb{F}_{p}^{k}$. Then:

$$
\sum_{\substack{t \in \mathbb{F}_{p}^{k}, \prod_{l=1}^{k}\left[f j_{j}(x, t) \neq 0\right.}} \frac{\left[d_{j_{1}, \ldots, j_{k}}\right]}{\prod_{l=1}^{k}[f]_{j_{l}}(x, t)}=\sum_{\substack{s \in \mathbb{F}_{p}^{k}, s_{1} \ldots k_{k} \neq 0}} \frac{\left[d_{j_{1}, \ldots, j_{k}}\right]}{s_{1} \ldots s_{k}}=\sum_{s \in \mathbb{F}_{p}^{k}}\left[d_{j_{1}, \ldots, j_{k}}\right]\left(s_{1} \ldots s_{k}\right)^{p-2}=0
$$

Lemma 4. The second sum in the right-hand side of Equation (55) equals $(-1)^{k}[I]_{j_{1}, \ldots, j_{k}}^{p-1, \ldots, p-1}(x)$.

Proof. The inequality of Equation (37) takes the form of Equation (53). Now the lemma follows from Theorem 6.

Theorem 9 is proved.

Remark 3. The inequality of Equation (53) holds if $n \leqslant 2 k+2$ independently of $p$.

\subsection{Counting on $\kappa$-Folded Covers}

Let $\kappa>2$ be a positive integer. As in Sections 4.1 and 5.2, we consider $n$ nonzero linear functions on $\mathbb{C}^{k}, g_{j}=b_{j}^{1} t_{1}+\cdots+b_{j}^{k} t_{k}, j \in J$, with integer coefficients $b_{j}^{i} \in \mathbb{Z}$. Let a prime integer $p$ be good with respect to $\left(g_{j}\right)_{j \in J}$ and $\kappa \mid(p-1)$.

Assume that all weights $\left(a_{j}\right)_{j \in J}$ are equal to $-(\kappa-1)$. Under these assumptions, consider the algebraic Equation (27) and differential Equation (30). To construct a solution of these equations, choose 
a master polynomial $\Phi(z, t)=\prod_{j \in J} f_{j}(z, t)^{(\kappa-1) \frac{p-1}{\kappa}}$, and consider the Taylor expansion at $t=0$ of the polynomial $F(z, t)$ in Equation (49) to obtain the solution $[I]^{p-1, \ldots, p-1}(z)$ of the algebraic Equation (27) and differential Equation (30) by taking the coefficient of $\left(t_{1} \ldots t_{k}\right)^{p-1}$ of the Taylor expansion (see Theorem 5 and Formula (50)).

Let $x=\left(x_{1}, \ldots, x_{n}\right) \in \mathbb{F}_{p}^{n}$. Let $\Gamma(x)$ be the affine hypersurface:

$$
y^{\kappa}=\prod_{j \in J}[f]_{j}(x, t)
$$

over $\mathbb{F}_{p}$ (cf. Section 5.2).

Theorem 10. Let a prime integer $p$ be good with respect to $\left(g_{j}\right)_{j \in J}$. Let $\kappa \mid(p-1)$ and $\kappa \neq p-1$. Let:

$$
n(\kappa-1) \frac{p-1}{\kappa}-k<(k+1)(p-1), \quad n(\kappa-2) \frac{p-2}{\kappa}-k<k(p-1)
$$

Then for any independent $\left\{j_{1}<\cdots<j_{k}\right\} \subset J$ we have:

$$
\int_{\Gamma(x)} \frac{\left[d_{j_{1}, \ldots, j_{k}}\right]}{\prod_{l=1}^{k}[f]_{j_{l}}(x, t)}=(-1)^{k}[I]_{j_{1}, \ldots, j_{k}}^{p-1, \ldots, p-1}(x)
$$

This theorem is a generalization of [3], Example 1.7 and Theorem 9.

Proof. It is easy to see that:

$$
\begin{aligned}
\int_{\Gamma(x)} \frac{\left[d_{j_{1}, \ldots, j_{k}}\right]}{\left.\prod_{l=1}^{k}[f]\right]_{j_{l}}(x, t)} & =\sum_{\prod_{l=1}^{k}[f]_{j_{l}}(x, t) \neq 0} \frac{\left[d_{j_{1}, \ldots, j_{k}}\right]}{\prod_{l=1}^{k}[f]_{j_{l}}(x, t)} \\
& +\sum_{\ell=1}^{\kappa-1} \sum_{t \in \mathbb{F}_{p}^{k}} \frac{\left[d_{j_{1}, \ldots, j_{k}}\right]}{\prod_{l=1}^{k}[f]_{j_{l}}(x, t)}\left(\prod_{j \in J}[f]_{j}(x, t)\right)^{\ell \frac{p-1}{\kappa}}
\end{aligned}
$$

The first sum on the right-hand side equals zero by Lemma 3.

Consider the Taylor expansion of the polynomial $\frac{\left[d_{j_{1}, \ldots j_{k}}\right]}{\prod_{l=1}^{k}[f]_{j_{l}}(x, t)}\left(\prod_{j \in J}[f]_{j}(x, t)\right)^{\ell \frac{p-1}{\kappa}}$. Consider the monomials of the form $t_{1}^{l_{1}(p-1)} \ldots t_{k}^{l_{k}(p-1)}$, where $l_{1}, \ldots, l_{k}$ are positive integers. If $\ell \leqslant \kappa-2$, the second inequality in Equation (57) implies that the coefficients of such monomials in the Taylor expansion are all equal to zero, and hence the sum $\sum_{t \in \mathbb{F}_{p}^{k}} \frac{\left[d_{j_{1} \ldots j_{k}}\right]}{\prod_{l=1}^{k}[f]_{j_{l}}(x, t)}\left(\prod_{j \in J}[f]_{j}(x, t)\right)^{\ell \frac{p-1}{\kappa}}$ equals zero for $\ell \leqslant \kappa-2$. If $\ell=\kappa-1$, the first inequality in Equation (57) implies that among the monomials of the form $t_{1}^{l_{1}(p-1)} \ldots t_{k}^{l_{k}(p-1)}$, only $t_{1}^{p-1} \ldots t_{k}^{p-1}$ may appear with a nonzero coefficient in the Taylor expansion. Hence:

$$
\sum_{t \in \mathbb{P}_{p}^{k}} \frac{\left[d_{j_{1}, \ldots, j_{k}}\right]}{\prod_{l=1}^{k}[f]_{j_{l}}(x, t)}\left(\prod_{j \in J}[f]_{j}(x, t)\right)^{(\kappa-1)^{\frac{p-1}{\kappa}}}=(-1)^{k}[I]_{j_{1}, \ldots, j_{k}}^{p-1, \ldots, p-1}(x)
$$

by Theorem 6 . The theorem is proved.

Remark 4. The inequalities of Equation (57) are implied by the system of inequalities:

$$
\frac{\kappa}{\kappa-1}(k+1) \geqslant n, \quad \frac{\kappa}{\kappa-2} k \geqslant n
$$

independently of $p$. If $k \geqslant \kappa-2$, then the inequality $\frac{\kappa}{\kappa-1}(k+1) \geqslant n$ implies inequality, $\frac{\kappa}{\kappa-2} k \geqslant n$, and is enough for Theorem 10 to hold. In particular, if $k \geqslant k-2$, then $n=k+2$ is admissible. 


\section{Bethe Ansatz}

The goal of the Bethe ansatz is to construct mutual eigenvectors of the geometric Hamiltonians $\left([K]_{i}(x)\right)_{i \in J}$ defined in Equation (29).

As in Sections 4.1, 5.2 and 5.3, we consider $n$ nonzero linear functions on $\mathbb{C}^{k}, g_{j}=b_{j}^{1} t_{1}+\cdots+$ $b_{j}^{k} t_{k}, j \in J$, with integer coefficients $b_{j}^{i} \in \mathbb{Z}$. Let a prime integer $p$ be good with respect to $\left(g_{j}\right)_{j \in J}$. Let $a=\left(a_{j}\right)_{j \in J}$ be nonzero integer weights $a_{j} \in \mathbb{Z}, a_{j} \neq 0$.

Recall the functions $[f]_{C}(z)=\sum_{i \in J}\left[\lambda_{C}^{i}\right] z_{i}$ with $C \in \mathfrak{C}_{\mathbb{F}_{p}}$. Assume that $x=\left(x_{1}, \ldots, x_{n}\right) \in \mathbb{F}_{p}^{n}$ is such that $f_{C}(x) \neq 0$ for any $C \in \mathfrak{C}_{\mathbb{F}_{p}}$. Then $\left(\left[K_{i}\right](x)\right)_{i \in J}$ are well-defined linear operators on $V_{\mathbb{F}_{p}}$.

Introduce the system of the Bethe ansatz equations:

$$
\sum_{j \in J} b_{j}^{i} \frac{\left[a_{j}\right]}{[f]_{j}(x, t)}=0, \quad i=1, \ldots, k
$$

with respect to the unknown $t=\left(t_{1}, \ldots, t_{k}\right) \in \mathbb{F}_{p}^{k}$.

Theorem 11. If $t^{0} \in \mathbb{F}_{p}^{k}$ is a solution of Equation (61), then the vector:

$$
F\left(x, t^{0}\right)=\sum_{\substack{\text { independent } \\\left\{j_{1}<\cdots<j_{k}\right\} \subset J}} \frac{\left[d_{j_{1}, \ldots, j_{k}}\right]}{[f]_{j_{1}}\left(x, t^{0}\right) \ldots[f]_{j_{k}}\left(x, t^{0}\right)} F\left(H_{j_{1}}, \ldots, H_{j_{k}}\right)
$$

satisfies Equation (27) and is an eigenvector of the geometric Hamiltonians:

$$
[K]_{i}(x) F\left(x, t^{0}\right)=\frac{\left[a_{i}\right]}{[f]_{i}\left(x, t^{0}\right)} F\left(x, t^{0}\right), \quad i=1, \ldots, n
$$

Proof. Equation (27) for $F\left(x, t^{0}\right)$ follows from Equations (20) and (21) reduced modulo $p$. Because $t^{0}$ is a solution of Equation (61), the left-hand side of Equation (21) equals zero. Equation (63) is a straightforward corollary of the key identity of Equation (28) (see the proof of [4], Theorem 2.1).

Corollary 3. Let $t^{0}$ and $t^{1}$ be distinct solutions of the Bethe ansatz Equation (61); then $[S]^{(a)}\left(F\left(x, t^{0}\right), F\left(x, t^{1}\right)\right)=0$.

Proof. Because $t^{0} \neq t^{1}$, there exists $i$ such that $[f]_{i}\left(x, t^{0}\right) \neq[f]_{i}\left(x, t^{1}\right)$. Hence $[K]_{i}(x)$ has distinct eigenvalues on $F\left(x, t^{0}\right), F\left(x, t^{1}\right)$, but $\left[K_{i}\right](x)$ is symmetric:

$$
[S]^{(a)}\left([K]_{i}(x) F\left(x, t^{0}\right), F\left(x, t^{1}\right)\right)=[S]^{(a)}\left(F\left(x, t^{0}\right),[K]_{i}(x) F\left(x, t^{1}\right)\right)
$$

Acknowledgments: This work was supported in part by NSF grants DMS-1362924, DMS-1665239.

Conflicts of Interest: The author declares no conflict of interest.

\section{References}

1. Manin, Y.I. The Hasse-Witt Matrix of an Algebraic Curve. Izv. Akad. Nauk SSSR Ser. Mat. 1961, 25, $153-172$. (In Russian)

2. Clemens, H.C. A Scrapbook of Complex Curve Theory, 2nd ed.; Graduate Studies in Mathematics; AMS: Providence, RI, USA, 2003.

3. Schechtman, V.; Varchenko, A. Solutions of KZ Differential Equations Modulo p. arXiv 2017, arXiv:1707.02615.

4. Varchenko, A. Remarks on the Gaudin Model Modulo p. arXiv 2017, arXiv:1708.06264. 
5. Schechtman, V.; Varchenko, A. Arrangements of Hyperplanes and Lie Algebra Homology. Invent. Math. 1991, 106, 139-194.

6. Bjorner, A. On the Homology of Geometric Lattices. Algebra Univ. 1984, 14, 107-128.

7. Varchenko, A.; Young, C. Cyclotomic discriminantal arrangements and diagram automorphisms of Lie algebras. arXiv 2016, arXiv:1603.07125.

8. Felder, G.; Markov, Y.; Tarasov, V.; Varchenko, A. Differential Equations Compatible with KZ equations. Math. Phys. Anal. Geom. 2000, 3, 139-177.

9. Varchenko, A. Bethe Ansatz for Arrangements of Hyperplanes and the Gaudin Model. Mosc. Math. J. 2006, 6, 195-210.

10. Varchenko, A. Quantum Integrable Model of an Arrangement of Hyperplanes. SIGMA 2011, 7, $55 \mathrm{p}, 032$.

11. Varchenko, A. Special Functions, KZ Type Equations, and Representation Theory; CBMS, Regional Conference Series in Mathematics, n. 98; AMS: Providence, RI, USA, 2003.

(C) 2017 by the author. Licensee MDPI, Basel, Switzerland. This article is an open access article distributed under the terms and conditions of the Creative Commons Attribution (CC BY) license (http://creativecommons.org/licenses/by/4.0/). 